\title{
Publisher Correction: A downy mildew effector evades recognition by polymorphism of expression and subcellular localization
}

\author{
Shuta Asai ${ }^{1,2}$, Oliver J. Furzer ${ }^{2,4}$, Volkan Cevik ${ }^{2,5}$, Dae Sung Kim²,6, Naveed Ishaque ${ }^{2,7}$, Sandra Goritschnig ${ }^{3}$, \\ Brian J. Staskawicz ${ }^{3}$, Ken Shirasu (1D ${ }^{1} \&$ Jonathan D.G. Jones ${ }^{2}$
}

Correction to: Nature Communications https://doi.org/10.1038/s41467-018-07469-3; published online 05 December 2018

The original version of this article contained an error in the author affiliations.

Oliver J. Furzer was incorrectly associated with Department of Plant Sciences, College of Life Sciences, Wuhan University, 430072, Wuhan, China.

This has now been corrected in the HTML version of the article. The PDF version of the article was correct at the time of publication.

Furthermore, the original version of this article stated that correspondence and requests for materials should be addressed to Heidelberg.Center.for.Personalized.Oncology, DKFZ-HIPO, DKFZ, Heidelberg 69120Germany S.A. (email: shuta.asai@riken.jp) or to J.D.G.J. (email: jonathan.jones@tsl.ac.uk). The words "Heidelberg.Center.for.Personalized.Oncology, DKFZ-HIPO, DKFZ, Heidelberg 69120Germany" were introduced inadvertently.

This has now been corrected in the PDF version of the article. The HTML version of the article was correct at the time of publication.

Published online: 08 January 2019

(i) Open Access This article is licensed under a Creative Commons Attribution 4.0 International License, which permits use, sharing, adaptation, distribution and reproduction in any medium or format, as long as you give appropriate credit to the original author(s) and the source, provide a link to the Creative Commons license, and indicate if changes were made. The images or other third party material in this article are included in the article's Creative Commons license, unless indicated otherwise in a credit line to the material. If material is not included in the article's Creative Commons license and your intended use is not permitted by statutory regulation or exceeds the permitted use, you will need to obtain permission directly from the copyright holder. To view a copy of this license, visit http://creativecommons.org/licenses/by/4.0/.

(C) The Author(s) 2019

\footnotetext{
${ }^{1}$ Center for Sustainable Resource Science, RIKEN, 1-7-22 Suehiro-cho, Tsurumi, Yokohama, Kanagawa 230-0045, Japan. ${ }^{2}$ The Sainsbury Laboratory, Norwich Research Park, Norwich NR4 7UH, UK. ${ }^{3}$ Department of Plant and Microbial Biology, University of California, Berkeley, CA 94720, USA. ${ }^{4}$ Present address: Department of Biology, University of North Carolina at Chapel Hill, Chapel Hill, NC 27599, USA. ${ }^{5}$ Present address: Department of Biology \& Biochemistry, University of Bath, Bath BA2 7AY, UK. ${ }^{6}$ Present address: Department of Plant Sciences, College of Life Sciences, Wuhan University, Wuhan 430072, China. ${ }^{7}$ Present address: Heidelberg Center for Personalized Oncology, DKFZ-HIPO, DKFZ, Heidelberg 69120, Germany. These authors contributed equally: Oliver J. Furzer, Volkan Cevik. The original article can be found online at https://doi.org/10.1038/s41467-018-07469-3. Correspondence and requests for materials should be addressed to S.A. (email: shuta.asai@riken.jp) or to J.D.G.J. (email: jonathan.jones@tsl.ac.uk)
} 